\title{
One-Photon and Two-Photon Double-Slit Interferences in Spontaneous and Stimulated Parametric Down-Conversions
}

\author{
De-Zhong Cao, ${ }^{1}$ Zhuan Li, ${ }^{1}$ Yan-Hua Zhai, ${ }^{1}$ and Kaige Wang $^{2,1, *}$ \\ ${ }^{1}$ Department of Physics, Applied Optics Beijing Area Major Laboratory, \\ Beijing Normal University, Beijing 100875, China \\ ${ }^{2}$ CCAST (World Laboratory), P. O. Box 8730, Beijing 100080, China
}

(Dated: November 2, 2018)

\begin{abstract}
We theoretically discuss one-photon and two-photon double-slit interferences for spontaneous and stimulated parametric down-conversions. We show that the two-photon sub-wavelength interference can exist in a general spontaneous parametric down-conversion (SPDC) for both type I and type II crystals. We propose an alternative way to observe sub-wavelength interference by a joint-intensity measurement which occurs for only type I crystal in a higher gain of SPDC. When a signal beam injects into the crystal, it may create two interference patterns by two stimulated down-converted beams, showing no sub-wavelength interference effect.
\end{abstract}

PACS numbers: 42.50.Dv, 42.65.Lm, 42.25.Hz, 42.82.Cr

\section{INTRODUCTION}

The Young's double-slit interference experiment is one of the powerful ways to exhibit the nature of optical field, both classical and nonclassical coherence effects. In the recent years, an interesting subject is devoted to the study of twophoton double-slit interference in the process of spontaneous parametric down-conversion (SPDC). [1]-[15] Since in this process a pair of converted beams created by a pump beam is in entanglement, the two-photon double-slit interference may show some peculiar phenomena such as the quantum sub-wavelength lithography and the ghost interference. For the former, both signal and idler beams are set together to pass through a double-slit, [5], 6], [8]- [1], 13], 14] and for the latter, the double-slit is placed on the path for only one beam. [1]- [4], [7], 12], 15] The original idea of the quantum lithography comes from the reduction of de Broglie wavelength for combining two massive particles. In optical system, the sub-wavelength interference occurs for a biphoton state. [16] In addition to the two-photon double-slit interference, the quantum lithography can be carried out in a Mach-Zehnder interferometer. 17]-[19] Due to the fact that this effect can overcome the Rayleigh diffraction limit, it may have prospective application in photo-lithography technology.

In most of the theoretical analyses, the sub-wavelength interference is explained by a two-photon entangled state which can be acquired in the SPDC with very low gain. Nevertheless, it is the obstacle in practical application due to the lower power. Therefore, the exploration of these quantum effects in macroscopic regime makes sense. [15], [18] In this paper, we study one-photon and two-photon double-slit interferences in both spontaneous and stimulated parametric down-conversions. We focus on the case in which a double-slit is inserted on the paths for both signal and idler beams. We find that the sub-wavelength lithography can occur at very high gain of SPDC with substantial visibility. The discussion covers both type I and type II crystals which exhibit different behaviors in two-photon interference. In the stimulated process, the amplified beam cannot perform quantum lithography but create rich interference patterns. The paper is organized as follows: in Sec. II we briefly review double-slit interferences for a coherent state and a two-photon state. In Sec. III we cite several formula as review of the optical parametric down-conversion process. We analyze two-photon double-slit interference in Secs. IV and V for the spontaneous and stimulated processes, respectively. The final section VI is the conclusion and discussion.

\section{DOUBLE-SLIT INTERFERENCES FOR A COHERENT STATE AND A TWO-PHOTON STATE}

We consider the scheme of Young's double-slit experiment as shown in Fig. 1. The double-slit function is defined by

$$
T(x)=\operatorname{rect}\left(\frac{x-d / 2}{b}\right)+\operatorname{rect}\left(\frac{x+d / 2}{b}\right),
$$

*Electronic address: wangkg@bnu.edu.cn 
where $d$ is the distance between the centers of two slits and $b$ is the width of each slit. In Fig. 1, both the double-slit and the detection screen are placed at the two focus planes of a lens. By ignoring the thickness of the double-slit, the transverse envelope operators of the input field $e(x, t)$ and the output field $e^{\prime}(x, t)$ of the double-slit are related as

$$
e^{\prime}(x, t)=T(x) e(x, t)+[1-T(x)] e_{v a c}(x, t),
$$

where the vacuum field operator $e_{v a c}(x, t)$ is introduced for the sake of $e^{\prime}(x, t)$ satisfying the bosonic commutation relation. Since the vacuum field has no contribution to the normal-order correlation, it can be neglected in the calculations below.

In the paraxial approximation, the field $r(x, t)$ in the detection plane $P_{2}$ is expressed by the Fourier transform of the lens

$$
r(x, t)=\sqrt{\frac{k}{2 \pi f}} \int e^{\prime}\left(x^{\prime}, t\right) \exp \left[-i \frac{k}{f} x^{\prime} x\right] d x^{\prime} .
$$

By substituting Eq. (2) into Eq. (3), one obtains

$$
r(x, t)=\frac{1}{2 \pi} \sqrt{\frac{k}{f}} \iint \widetilde{T}\left(\frac{k x}{f}-q\right) \widetilde{e}(q, \Omega) \exp [-i \Omega t] d q d \Omega,
$$

where

$$
\widetilde{T}(q)=\frac{1}{\sqrt{2 \pi}} \int T(x) e^{-i q x} d x=\frac{2 b}{\sqrt{2 \pi}} \operatorname{sinc}(q b / 2) \cos (q d / 2)
$$

is the Fourier transform of the double-slit function $T(x)$, and $\widetilde{e}(q, \Omega)$ is the Fourier transform of $e(x, t)$ for both the spatial and temporal variables.

First, we consider the input field to be a stationary, monochromatic plane wave in a coherent state

$$
\langle e(x, t)\rangle=A
$$

where $A$ is a constant. It has

$$
\langle\widetilde{e}(q, \Omega)\rangle=2 \pi A \delta(q) \delta(\Omega) .
$$

In the detection plane, the first-order correlation is calculated as

$$
G^{(1)}\left(x_{1}, x_{2}, t\right) \equiv\left\langle r^{\dagger}\left(x_{1}, t\right) r\left(x_{2}, t\right)\right\rangle=\frac{k A^{2}}{f} \widetilde{T}^{*}\left(\frac{k x_{1}}{f}\right) \widetilde{T}\left(\frac{k x_{2}}{f}\right) .
$$

Hence, the intensity distribution in the detection plane is written as

$$
G^{(1)}(x, x, t) \equiv\left\langle r^{\dagger}(x, t) r(x, t)\right\rangle=\frac{k A^{2}}{f} \widetilde{T}^{2}\left(\frac{k x}{f}\right)=I_{0} \operatorname{sinc}^{2}\left(\frac{\pi b x}{\lambda f}\right) \cos ^{2}\left(\frac{\pi d x}{\lambda f}\right)
$$

where $I_{0}=\frac{2 k b^{2} A^{2}}{\pi f}$ and $\lambda=2 \pi / k$. Note that $\widetilde{T}(x)$ is a real function. Equation (9) represents an interference fringe with the interval $\lambda f / d$ in the range $\lambda f / b$, as shown in Fig. 2.

Similarly, the second-order correlation function can be obtained as

$$
\begin{aligned}
G^{(2)}\left(x_{1}, x_{2}, t\right) & =\left\langle r^{\dagger}\left(x_{1}, t\right) r^{\dagger}\left(x_{2}, t\right) r\left(x_{2}, t\right) r\left(x_{1}, t\right)\right\rangle \\
& =\frac{k^{2} A^{4}}{f^{2}} \widetilde{T}^{2}\left(\frac{k x_{1}}{f}\right) \widetilde{T}^{2}\left(\frac{k x_{2}}{f}\right) .
\end{aligned}
$$

According to the theory of field coherence, the separability of spatial variables in the first-order and the second-order correlation functions verifies the perfect coherence of the field. Since the field operators at different positions are commutable, the second-order correlation of the field is in fact the spatial intensity correlation and it can be observed by a coincidence measurement as shown in Fig. 1a. The spatial patterns related to $G^{(1)}(x, x, t)$ and $G^{(2)}\left(x_{1}, x_{2}, t\right)$ are called the one-photon and the two-photon interferences, respectively. According to Eq. (10), in the coincidence measurement, if we scan one detector by fixing another, the same interference fringe as the one-photon interference 
can be observed. Now, we introduce two other ways of observations for the two-photon double-slit interference. One is the spatial intensity-correlation measurement by scanning two detectors synchronously at a pair of symmetric positions, $x_{1}=-x_{2}=x$. The other one is the two-photon intensity measurement by using a two-photon detector which generates an photo-electron by absorbing two photons. Applying these two observations to Eq. (10), one has

$$
G^{(2)}(x, x, t)=G^{(2)}(x,-x, t)=I_{0}^{2} \operatorname{sinc}^{4}\left(\frac{\pi b x}{\lambda f}\right) \cos ^{4}\left(\frac{\pi d x}{\lambda f}\right) .
$$

In Fig. 2, we plot $G^{(2)}(x, x, t)\left(G^{(2)}(x,-x, t)\right)$ in comparison with $G^{(1)}(x, t)$ for the coherent beam. It shows that the two interference patterns are alike.

The above discussion on the coherent state is analogous to the classical field. Then, we consider a two-photon state as input, which is the quantum state without classical analogue. A general two-photon state can be written as

$$
|\psi\rangle=\int d q_{s} d q_{i} C\left(q_{s}, q_{i}\right) a_{s}^{\dagger}\left(q_{s}\right) a_{i}^{\dagger}\left(q_{i}\right)|0\rangle
$$

where $a_{s}^{\dagger}$ and $a_{i}^{\dagger}$ are the creation operators for $s$ and $i$ photons which are assumed to be distinguishable. $q_{s}$ and $q_{i}$ are the transverse wave-vectors. When the input field is stationary, Eq. (4) can be simplified as

$$
r(x)=\sqrt{\frac{k}{2 \pi f}} \int \widetilde{T}\left(\frac{k x}{f}-q\right) \widetilde{e}(q) d q .
$$

By using Eq. (13), the first-order correlation functions for $s$-photon and $i$-photon in the detection plane are obtained as

$$
\begin{aligned}
G_{s}^{(1)}\left(x_{1}, x_{2}\right) & =\frac{k}{2 \pi f} \int d q d q_{1} d q_{2} C^{*}\left(q_{1}, q\right) C\left(q_{2}, q\right) \widetilde{T}^{*}\left(\frac{k x_{1}}{f}-q_{1}\right) \widetilde{T}\left(\frac{k x_{2}}{f}-q_{2}\right) \\
G_{i}^{(1)}\left(x_{1}, x_{2}\right) & =\frac{k}{2 \pi f} \int d q d q_{1} d q_{2} C^{*}\left(q, q_{1}\right) C\left(q, q_{2}\right) \widetilde{T}^{*}\left(\frac{k x_{1}}{f}-q_{1}\right) \widetilde{T}\left(\frac{k x_{2}}{f}-q_{2}\right)
\end{aligned}
$$

respectively.

For two fields case, the second-order correlation function is defined by

$$
G^{(2)}\left(x_{1}, x_{2}, t\right)=\left\langle r_{i}^{\dagger}\left(x_{1}, t\right) r_{s}^{\dagger}\left(x_{2}, t\right) r_{s}\left(x_{2}, t\right) r_{i}\left(x_{1}, t\right)\right\rangle
$$

which describes the coincidence probability of $s$-photon at position $x_{2}$ and $i$-photon at position $x_{1}$. In the case of $x_{1}=x_{2}=x$, it describes a two-photon intensity distribution. First, for the two-photon state (12), we calculate the two-photon wavepacket in the detection plane

$$
\left\langle 0\left|r_{s}\left(x_{2}\right) r_{i}\left(x_{1}\right)\right| \psi\right\rangle=\frac{k}{2 \pi f} \int d q_{s} d q_{i} C\left(q_{s}, q_{i}\right) \widetilde{T}\left(\frac{k x_{2}}{f}-q_{s}\right) \widetilde{T}\left(\frac{k x_{1}}{f}-q_{i}\right) .
$$

Then the second-order correlation is obtained as

$$
G^{(2)}\left(x_{1}, x_{2}\right)=\left|\left\langle 0\left|r_{s}\left(x_{2}\right) r_{i}\left(x_{1}\right)\right| \psi\right\rangle\right|^{2}
$$

We discuss two extreme cases: two photons are independent and perfectly entangled in the transverse wave-vector. In the unentangled case, $C\left(q_{s}, q_{i}\right)=C_{s}\left(q_{s}\right) C_{i}\left(q_{i}\right)$, the first- and second-order correlations are written as

$$
\begin{aligned}
G_{m}^{(1)}\left(x_{1}, x_{2}\right) & =\frac{k}{2 \pi f} \int d q C_{m}^{*}(q) \widetilde{T}^{*}\left(\frac{k x_{1}}{f}-q\right) \int d q C_{m}(q) \widetilde{T}\left(\frac{k x_{2}}{f}-q\right), \quad(m=s, i) \\
G^{(2)}\left(x_{1}, x_{2}\right) & =\left|\frac{k}{2 \pi f} \int d q C_{s}(q) \widetilde{T}\left(\frac{k x_{2}}{f}-q\right) \int d q C_{i}(q) \widetilde{T}\left(\frac{k x_{1}}{f}-q\right)\right|^{2} \\
& =G_{i}^{(1)}\left(x_{1}, x_{1}\right) G_{s}^{(1)}\left(x_{2}, x_{2}\right) .
\end{aligned}
$$

Equations (18) show two separabilities. The one is the separability of positions in both the first- and second-order correlation functions. The other is that the second-order correlation is factorized to two one-photon intensity distribution. That is, the two-photon interference consists of two individual one-photon interferences, verifying the 
Dirac's statement: "Each photon interferes only with itself. Interference between two different photons never occurs." However, these features mean the perfect first- and second-order coherencies for the two independent photons.

In the opposite extreme, the perfect entanglement in wavevector, $q_{s}+q_{i}=0$, is hold in state (12). For simplicity, we assume $C\left(q_{s}, q_{i}\right) \rightarrow \delta\left(q_{s}+q_{i}\right)$, and the first- and second-order correlations are written as

$$
\begin{aligned}
G_{s}^{(1)}\left(x_{1}, x_{2}\right) & =G_{i}^{(1)}\left(x_{1}, x_{2}\right)=\frac{k}{2 \pi f} \int d q \widetilde{T}^{*}\left(\frac{k x_{1}}{f}+q\right) \widetilde{T}\left(\frac{k x_{2}}{f}+q\right)=\frac{k}{f \sqrt{2 \pi}} \widetilde{T}\left[\frac{k}{f}\left(x_{2}-x_{1}\right)\right], \\
G^{(2)}\left(x_{1}, x_{2}\right) & =\left|\frac{k}{2 \pi f} \int d q \widetilde{T}\left(\frac{k x_{2}}{f}+q\right) \widetilde{T}\left(\frac{k x_{1}}{f}-q\right)\right|^{2}=\frac{k^{2}}{2 \pi f^{2}} \widetilde{T}^{2}\left[\frac{k}{f}\left(x_{1}+x_{2}\right)\right],
\end{aligned}
$$

where we use the integrals

$$
\begin{aligned}
\int d q \widetilde{T}^{*}\left(\frac{k x_{1}}{f} \pm q\right) \widetilde{T}\left(\frac{k x_{2}}{f} \pm q\right) & =\frac{1}{2 \pi} \iiint d q d x_{1}^{\prime} d x_{2}^{\prime} T\left(x_{1}^{\prime}\right) T\left(x_{2}^{\prime}\right) e^{i\left(\frac{k x_{1}}{f} \pm q\right) x_{1}^{\prime}-i\left(\frac{k x_{2}}{f} \pm q\right) x_{2}^{\prime}} \\
& =\iint d x_{1}^{\prime} d x_{2}^{\prime} T\left(x_{1}^{\prime}\right) T\left(x_{2}^{\prime}\right) \delta\left(x_{1}^{\prime}-x_{2}^{\prime}\right) e^{i \frac{k x_{1}}{f} x_{1}^{\prime}-i \frac{k x_{2}}{f} x_{2}^{\prime}} \\
& =\int d x_{1}^{\prime} T\left(x_{1}^{\prime}\right) T\left(x_{1}^{\prime}\right) e^{i \frac{k}{f}\left(x_{1}-x_{2}\right) x_{1}^{\prime}}=\sqrt{2 \pi} \widetilde{T}\left[\frac{k}{f}\left(x_{2}-x_{1}\right)\right]
\end{aligned}
$$

and

$$
\int d q \widetilde{T}^{*}\left(\frac{k x_{1}}{f} \pm q\right) \widetilde{T}\left(\frac{k x_{2}}{f} \mp q\right)=\sqrt{2 \pi} \widetilde{T}\left[\frac{k}{f}\left(x_{2}+x_{1}\right)\right]
$$

Note that $T^{2}(x)=T(x)$. Equations (19) show that for the wavevector-entangled two-photon state the first- and second-order correlation functions in double-slit interference have position-correlation which results in decoherence. In the measurement, letting $x_{1}=x_{2}$ in Eq. (19), one obtains

$$
\begin{aligned}
G_{s}^{(1)}(x, x) & =G_{i}^{(1)}(x, x)=\frac{k}{f \sqrt{2 \pi}} \widetilde{T}[0], \\
G^{(2)}(x, x) & =\frac{k^{2}}{2 \pi f^{2}} \widetilde{T}^{2}\left[\frac{k}{f}(2 x)\right] .
\end{aligned}
$$

Therefore, the one-photon double-slit interference disappears completely and the two-photon double-slit interference shows a sub-wavelength property since it has

$$
\widetilde{T}^{2}\left(\frac{k}{f} 2 x\right) \propto \operatorname{sinc}^{2}\left[\frac{\pi b x}{(\lambda / 2) f}\right] \cos ^{2}\left[\frac{\pi d x}{(\lambda / 2) f}\right] .
$$

The fringe is the same as the ordinary double-slit interference with the half of the wavelength. The above analysis explains the complementarity of coherence and entanglement. [8], 10], 11] We emphasize that the discussion is also true for the case when $s$ and $i$ photons can be indistinguishable.

\section{THE BASIC FORMULA IN THE OPTICAL PARAMETRIC DOWN-CONVERSION}

In the optical parametric down-conversion, in which a plane-wave pump field of frequency $\omega_{p}$ activates a $\chi^{(2)}$ nonlinear crystal, the basic unitary transformation is described by [15], 20]-[22]

$$
\widetilde{e}_{m}(q, \Omega)=U_{m}(q, \Omega) \widetilde{a}_{m}(q, \Omega)+V_{m}(q, \Omega) \widetilde{a}_{n}^{\dagger}(-q,-\Omega) \quad(m \neq n=s, i),
$$

where $\widetilde{e}_{m}(q, \Omega)$ and $\widetilde{a}_{m}(q, \Omega)$ are the output and input field operators, respectively. $q$ is the transverse wavevector and $\Omega$ is the frequency deviation from the carrier frequency. The transfer coefficients $U_{m}(q, \Omega)$ and $V_{m}(q, \Omega)$ are given by 22$]$

$$
\begin{gathered}
U_{s}(q, \Omega)=\Theta_{s}(q, \Omega)\left[\cosh \Gamma(q, \Omega)+i \frac{\Delta(q, \Omega)}{2 \Gamma(q, \Omega)} \sinh \Gamma(q, \Omega)\right] \\
V_{s}(q, \Omega)=\Theta_{s}(q, \Omega) \frac{g}{\Gamma(q, \Omega)} \sinh \Gamma(q, \Omega)
\end{gathered}
$$




$$
\begin{gathered}
U_{i}(q, \Omega)=\Theta_{i}(q, \Omega)\left[\cosh \Gamma(-q,-\Omega)+i \frac{\Delta(-q,-\Omega)}{2 \Gamma(-q,-\Omega)} \sinh \Gamma(-q,-\Omega)\right] \\
V_{i}(q, \Omega)=\Theta_{i}(q, \Omega) \frac{g}{\Gamma(-q,-\Omega)} \sinh \Gamma(-q,-\Omega),
\end{gathered}
$$

where

$$
\begin{gathered}
\Theta_{m}(q, \Omega)=e^{i\left[k_{m z}(q, \Omega)-k_{n z}(-q,-\Omega)-2 k_{m}+k_{p}\right] l_{c} / 2}, \quad(m \neq n=s, i) \\
\Gamma(q, \Omega)=\sqrt{g^{2}-\Delta^{2}(q, \Omega) / 4} \\
\Delta(q, \Omega)=\left[k_{s z}(q, \Omega)+k_{i z}(-q,-\Omega)-k_{p}\right] l_{c} \\
\Delta_{0}=\left(k_{s}+k_{i}-k_{p}\right) l_{c}
\end{gathered}
$$

$g$ is the coupling strength and $l_{c}$ is the length of crystal. $\Delta_{0}$ is the collinear phase mismatching of the central frequency components which correspond to the wave-numbers $k_{j}(j=s, i, p)$. For simplicity, we assume that two down-converted beams have the degenerate carrier frequency $\omega_{p} / 2$. Hence, Eq. (31) can be reduced to an even function of both $q$ and $\Omega$

$$
\Delta(q, \Omega) \approx \Delta_{0}+\Omega^{2} / \Omega_{0}^{2}-q^{2} / q_{0}^{2}
$$

where $\Omega_{0}$ and $q_{0}$ are defined as the frequency and spatial-frequency bandwidths, respectively.

Equations (24)-(33) describe the SPDC process of type II crystal but also can be applicable to type I crystal. For the former, two converted beams are orthogonally polarized, whereas for the latter, they are degenerate in both polarization and frequency but spatially separated. Therefore, Eq. 24) can describe type I crystal by omitting the subscripts. As a matter of fact, under the assumption of the carrier frequency degeneracy, Eqs. (25) and (26) are the same as Eqs. (27) and (28).

\section{DOUBLE-SLIT INTERFERENCE IN SPONTANEOUS PARAMETRIC DOWN-CONVERSION}

In Fig. 1, the two down-converted beams generated from the crystal illuminate a double-slit and then are detected in the focus plane of the lens. We designate $a_{m}(x, t), e_{m}(x, t), e_{m}^{\prime}(x, t), r_{m}(x, t)$ the slowly varying field operators for the input surface $P_{i n}$ and the output surface $P_{\text {out }}$ of the crystal and the output plane of the double-slit $P_{1}$ and the detection plane $P_{2}$, respectively. Substituting Eq. (24) into Eq. (44), we may calculate the first- and the second-order correlations for the field in the detection plane $P_{2}$.

In this section, we consider the case of the spontaneous parametric down-conversion in which the input fields are in the vacuum state. The first-order correlations for two beams are obtained to be

$$
\begin{aligned}
G_{m}^{(1)}\left(x_{1}, x_{2}\right) & =M_{m}\left(x_{1}, x_{2}\right) \equiv\left\langle 0\left|r_{m}^{\dagger}\left(x_{1}, t\right) r_{m}\left(x_{2}, t\right)\right| 0\right\rangle \\
& =\frac{k / f}{(2 \pi)^{2}} \iint d q d \Omega \quad\left|V_{m}(q, \Omega)\right|^{2} \widetilde{T}^{*}\left(\frac{k x_{1}}{f}-q\right) \widetilde{T}\left(\frac{k x_{2}}{f}-q\right) .
\end{aligned} \quad(m=s, i)
$$

$G_{m}^{(1)}(x, x)$ illustrates the intensity distribution for beam $m$ in the detection plane. Then we consider the second-order correlation function defined by Eq. (15), which now describes the intensity correlation between the signal beam at position $x_{2}$ and the idler beam at position $x_{1}$. In the case of $x_{1}=x_{2}=x$, it describes a two-photon intensity distribution. For type II crystal, the two-photon intensity distribution can be measured experimentally by the scheme shown in Fig. 1b in which the coincidence measurement of two orthogonally polarized photons is performed at the same position $x_{1}=x_{2}=x$. However, for type I crystal, the subscripts $s$ and $i$ should be omitted in Eq. (15). If there is a two-photon detector available, one may observe the two-photon intensity distribution at position $x_{1}=x_{2}=x$. 
Alternatively, a realistic detective scheme for type I crystal is shown in Fig. 1a in which the intensity correlation is measured by two one-photon detectors at the different positions $x_{1}$ and $x_{2}$.

For the vacuum state set in Eq. (15), by using Eqs. (4) and (24), the second-order correlation is calculated as

$$
G^{(2)}\left(x_{1}, x_{2}\right)=M_{i}\left(x_{1}, x_{1}\right) M_{s}\left(x_{2}, x_{2}\right)+\left|N_{i s}\left(x_{1}, x_{2}\right)\right|^{2}+\delta_{i s}\left|M\left(x_{1}, x_{2}\right)\right|^{2},
$$

where $\delta_{i s}$ is 1 for type I and 0 for type II crystal. $M_{m}\left(x_{1}, x_{2}\right)$ is given by Eq. (34), and

$$
N_{m n}\left(x_{1}, x_{2}\right)=\frac{k / f}{(2 \pi)^{2}} \iint d q d \Omega \quad V_{m}(q, \Omega) U_{n}(-q,-\Omega) \widetilde{T}\left(\frac{k x_{1}}{f}-q\right) \widetilde{T}\left(\frac{k x_{2}}{f}+q\right) . \quad(m \neq n=s, i)
$$

Equation (35) shows that the second-order correlation is related to the first-order correlation. In Eq. (35), the first term, which is separable in term of both polarization and position, describes the part of two-photon interference contributed by two individual single-photon double-slit processes. However, the second and the third terms describe two-photon interference effect related to photon entanglement. The difference between the two types of crystals is devoted by the third term, that is, the first-order correlation $\left|M\left(x_{1}, x_{2}\right)\right|^{2}$.

We note that, since $\Theta_{i}(q, \Omega) \Theta_{s}(-q,-\Omega)=\Theta_{s}(q, \Omega) \Theta_{i}(-q,-\Omega)=\exp \left[-i \Delta_{0}\right]$, it has $V_{i}(q, \Omega) U_{s}(-q,-\Omega)=$ $V_{s}(q, \Omega) U_{i}(-q,-\Omega)$, and hence $N_{i s}\left(x_{1}, x_{2}\right)=N_{s i}\left(x_{1}, x_{2}\right)$. This result is obvious because exchange of the indices $i$ and $s$ makes no difference physically. Moreover, due to Eq. (33), $\Delta(q, \Omega)$ is the even function of $q$ and $\Omega$ under the assumption of the frequency degeneracy so that $\left|V_{i}(q, \Omega)\right|^{2}=\left|V_{s}(q, \Omega)\right|^{2}$. Therefore, two first-order correlation functions for the signal and the idler beams are equal in type II crystal, i.e. $M_{i}\left(x_{1}, x_{2}\right)=M_{s}\left(x_{1}, x_{2}\right)$. Nevertheless, we keep the subscripts in Eq. (35) for a general description of type II crystal in case the two converted beams have different carrier frequencies.

In order to obtain the analytical result for the integrals, we discuss two bandwidth limits of SPDC process: the broad and the narrow bandwidths. In the broadband limit, $q_{0} \gg 2 \pi / d, U_{m}(q, \Omega)$ and $V_{m}(q, \Omega)$ are much flatter in comparison with $\widetilde{T}(q)$ and one can set $U_{m}(q, \Omega) \approx U_{m}(0, \Omega)$ and $V_{m}(q, \Omega) \approx V_{m}(0, \Omega)$ in the integrals. By taking into account Eqs. (20) and (21), Eqs. (34) and (36) can be rewritten as

$$
M_{m}\left(x_{1}, x_{2}\right)=\frac{\eta_{m}}{\sqrt{2 \pi}} \int d q \widetilde{T}^{*}\left(\frac{k x_{1}}{f}-q\right) \widetilde{T}\left(\frac{k x_{2}}{f}-q\right)=\eta_{m} \widetilde{T}\left[\frac{k}{f}\left(x_{2}-x_{1}\right)\right], \quad(m=s, i),
$$

and

$$
N_{m n}\left(x_{1}, x_{2}\right)=\xi_{m n} \widetilde{T}\left[\frac{k}{f}\left(x_{1}+x_{2}\right)\right], \quad(m \neq n=s, i),
$$

respectively, where we define $\eta_{m}=\frac{k / f}{(2 \pi)^{3 / 2}} \int\left|V_{m}(0, \Omega)\right|^{2} d \Omega$ and $\xi_{m n}=\frac{k / f}{(2 \pi)^{3 / 2}} \int V_{m}(0, \Omega) U_{n}(0,-\Omega) d \Omega$.

In the broadband limit, which exhibits the maximum entanglement in transverse wavevector for two converted beam, we see again the position-correlation in the correlation functions. The first-order correlation Eq. (37a) shows the same position-correlation as Eq. (19a) for the two-photon state with the maximum wavevector-entanglement. This makes the one-photon intensity distribution in the detection plane $P_{2}$ homogeneous, $M_{m}(x, x)=\eta_{m} \widetilde{T}(0)$, so that the one-photon double-slit interference disappears completely.

In this limit, the second-order correlation (35) is obtained to be

$$
G^{(2)}\left(x_{1}, x_{2}\right)=\eta_{i} \eta_{s}\left\{\widetilde{T}^{2}(0)+\delta_{i s} \widetilde{T}^{2}\left[\frac{k}{f}\left(x_{2}-x_{1}\right)\right]\right\}+\left|\xi_{i s}\right|^{2} \widetilde{T}^{2}\left[\frac{k}{f}\left(x_{1}+x_{2}\right)\right] .
$$

The first term in \{\} comes from two individual single-photon double-slit processes which are now homogeneous. The second term in \{\} and the last term manifest explicitly the position-corrlation. If we fix one detector at a position and scan another in the coincidence measurement, the interference fringe observed is the same as the single-photon one. To show the position correlation in the two-photon interference, we discuss two special observations, that is, $x_{1}=x_{2}=x$ and $x_{1}=-x_{2}=x$. Equation (39) is reduced to

$$
G^{(2)}(x, x)=\eta_{i} \eta_{s}\left(1+\delta_{i s}\right) \widetilde{T}^{2}(0)+\left|\xi_{i s}\right|^{2} \widetilde{T}^{2}\left(\frac{k}{f} 2 x\right),
$$

and

$$
G^{(2)}(x,-x)=\left(\eta_{i} \eta_{s}+\left|\xi_{i s}\right|^{2}\right) \widetilde{T}^{2}(0)+\delta_{i s} \eta_{i} \eta_{s} \widetilde{T}^{2}\left(\frac{k}{f} 2 x\right) .
$$


The former exhibits a two-photon intensity distribution and the latter exhibits the intensity correlation at a pair of symmetric positions. Both Eqs. (40) and (41) include a term $\widetilde{T}^{2}\left(\frac{k}{f} 2 x\right)$ which characterizes a sub-wavelength interference pattern by the factor of $\lambda / 2$ in comparison with the ordinary interference shown by Eq. (9). Obviously, due to Eq. (40), the sub-wavelength interference for the two-photon intensity distribution can occur in both type I and type II crystals. However, Eq. (41) shows that, for type I crystal, when a pair of single-photon detectors are placed at a pair of symmetric positions and moved synchronously in the opposite direction, the sub-wavelength interference can be also observed. But this effect never happens in type II crystal.

According to Eqs. (40) and (41), the visibilities of fringes designated by $G^{(2)}(x, x)$ and $G^{(2)}(x,-x)$ are calculated to be

$$
\mathcal{V}_{1}=\frac{1}{1+2\left(1+\delta_{i s}\right) \theta}
$$

and

$$
\mathcal{V}_{2}=\frac{1}{3+2 / \theta}
$$

respectively, where $\theta \equiv \eta_{i} \eta_{s} /\left|\xi_{i s}\right|^{2}$. Note that $\mathcal{V}_{2}$ makes sense only for type I crystal, for which $\theta \equiv \eta^{2} /|\xi|^{2}$. As the parameter $\theta$ is increased from a very small quantity, $\mathcal{V}_{1}$ decreases monotonously from unity and $\mathcal{V}_{2}$ increases from zero up to $1 / 3$. Since the parameter $\theta$ is related to the coupling strength $g$ of SPDC, we plot the visibilities as functions of $g$ in Figs. 3, in which $\mathcal{V}_{1}$ s for type II and type I crystals are indicated by the solid and dashed lines, respectively, and $\mathcal{V}_{2}$ for type I crystal is indicated by the dotted line. In a weak coupling of SPDC, which may generate approximately a two-photon entangled state, the visibilities $\mathcal{V}_{1}$ for both type I and II crystals reach perfectness. The sub-wavelength interference in the weak SPDC has been observed experimentally. [5], [9], 13], 19] The important fact is that, in Figs. 3 , the sub-wavelength interference can still exist, with a substantial visibility, even in very high gain of SPDC, in which the beams contain a large amount of photons. On the other hand, in the strong coupling of type I crystal, the sub-wavelength interference can occur in the joint-intensity measurement at a pair of symmetric positions. Equations (42) and (43) show that, for type I crystal, two observations of sub-wavelength interferences compete for visibility. When $\mathcal{V}_{1}$ reaches the perfectness, $\mathcal{V}_{2}$ vanishes. However, in the very high gain of SPDC of type I, two visibilities are the same as $25 \%$.

In the opposite limit, we assume that the SPDC has a very narrow bandwidth $q_{0} \ll 2 \pi / d$. Extremely, letting $q_{0} \rightarrow 0$, the transfer coefficient $V_{m}(q, \Omega)(m=s, i)$ tends to the delta function

$$
V_{m}(q, \Omega) \rightarrow V_{m}(0, \Omega) \delta(q)
$$

Equations (34) and (36) are respectively written as

$$
M_{m}\left(x_{1}, x_{2}\right)=\frac{1}{\sqrt{2 \pi}} \eta_{m} \widetilde{T}\left(\frac{k x_{1}}{f}\right) \widetilde{T}^{\star}\left(\frac{k x_{2}}{f}\right),
$$

and

$$
N_{m n}\left(x_{1}, x_{2}\right)=\frac{1}{\sqrt{2 \pi}} \xi_{m n} \widetilde{T}\left(\frac{k x_{1}}{f}\right) \widetilde{T}\left(\frac{k x_{2}}{f}\right) .
$$

In this limit, the position-correlation disappears completely. The second-order correlation is then

$$
G^{(2)}\left(x_{1}, x_{2}\right)=\frac{1}{2 \pi}\left[\left(1+\delta_{i s}\right) \eta_{i} \eta_{s}+\left|\xi_{i s}\right|^{2}\right] \widetilde{T}^{2}\left(\frac{k x_{1}}{f}\right) \widetilde{T}^{2}\left(\frac{k x_{2}}{f}\right) .
$$

Therefore, the one-photon intensity distribution $M_{m}(x, x)$ and the second-order correlation $G^{(2)}\left(x_{1}, x_{2}\right)$ in the plane $P_{2}$ are the same as the case for the coherent state. In the narrow bandwidth limit, since each converted beam is monochromatic, the one-photon double-slit interference occurs with the perfect visibility. On the other hand, two monochromatic converted beams have no more correlation in the transverse wavevector so that the position-correlation degrades completely in the two-photon interference.

We plot the two-photon interference patterns by varying the bandwidth of SPDC process $q_{0}$ in Figs. 4, in which Figs. 4a and 4b (4c and 4d) are for type I (type II) crystal. In Figs. 4a and 4c, a low gain of SPDC, $g=(1 / 2) \log 1.5$ (with the amplification rate 1.5) is taken in two-photon intensity measurement so that the sub-wavelength interferences with a better visibility are achieved when the normalized bandwidth $q_{0} b /(2 \pi)$ is increased. Two sets of patterns are very similar with exception of tiny higher intensity for type I crystal. Figures $4 \mathrm{~b}$ and $4 \mathrm{~d}$ show the interference patterns 
for the joint-intensity measurement of two one-photon detectors at a pair symmetric positions. The sub-wavelength interferences can be observed only for type I crystal when the gain of SPDC is higher, for instance, $g=(1 / 2) \log 10$ (with the amplification rate 10) is taken in the figures. Though the visibilities are lower, the intensities of the patterns are getting much higher. This also happens in the case of two-photon intensity measurement. The three plots of Figs. 4a-4c show that the bandwidth of SPDC governs two-photon sub-wavelength interference. For a very small bandwidth of SPDC, the two converted beams are de-entangled in transverse wavevector so that the nonclassical sub-wavelength interference disappears.

\section{DOUBLE-SLIT INTERFERENCE IN STIMULATED PARAMETRIC DOWN-CONVERSION}

In the stimulated optical parametric process, a signal beam is injected into the nonlinear crystal and is then amplified. The nonlinear crystal becomes an optical parametric amplifier (OPA). We assume inputting a stable plane-wave beam in a coherent state

$$
\left\langle\widetilde{a}_{s}(q, \Omega)\right\rangle=2 \pi A \delta(q-Q) \delta(\Omega),
$$

where $Q$ designates the transverse wavevector of the input beam deviated from the normal incidence. For type II crystal, we set the input beam as the signal which can be identified by the polarization, while the idler beam is in the vacuum state. For type I crystal, the subscript $s$ in the input beam (48) is omitted. Considering the input beam describing by Eq. (48) instead of the vacuum state, we calculate the first-order correlation in the plane $P_{2}$

$$
G_{m}^{(1)}\left(x_{1}, x_{2}\right)=W_{m}^{*}\left(x_{1}, Q\right) W_{m}\left(x_{2}, Q\right)+M_{m}\left(x_{1}, x_{2}\right), \quad(m=s, i)
$$

where

$$
\begin{aligned}
& W_{s}(x, Q)=A \sqrt{k / f} U_{s}(Q, 0) \widetilde{T}(k x / f-Q) \\
& W_{i}(x, Q)=A \sqrt{k / f} V_{i}(-Q, 0) \widetilde{T}(k x / f+Q)
\end{aligned}
$$

for type II crystal, and

$$
W(x, Q)=A \sqrt{k / f}[U(Q, 0) \widetilde{T}(k x / f-Q)+V(-Q, 0) \widetilde{T}(k x / f+Q)]
$$

for type I crystal. $M_{m}\left(x_{1}, x_{2}\right)$ has been defined by Eq. (34). In Eq. (49), the first and the second terms show contributions coming from the stimulated and the spontaneous processes, respectively, and they are independent in the one-photon interference pattern. Obviously, the stimulated part shows the first-order coherence due to the separability of the spatial variables. Nevertheless, for the input beam is enough strong, the spontaneous process can be neglected. $G_{m}^{(1)}(x, x) \simeq\left|W_{m}(x, Q)\right|^{2}$ describes an amplified double-slit interference pattern in comparison with the case without crystal. For type II crystal, the input signal beam creates two interference patterns: one for the signal beam with the amplification rate $\left|U_{s}(Q, 0)\right|^{2}$ and the other for the idler beam with the amplification rate $\left|V_{s}(-Q, 0)\right|^{2}$.24] According to Eqs. (50), these two patterns are the same as the ordinary fringe (see Eq. (92) and can be identified by the polarization and separated in space when the input beam is well tilted in incidence. However, for type I crystal, the one-photon interference pattern is written as

$$
\begin{aligned}
|W(x, Q)|^{2}= & \frac{k A^{2}}{f}\left\{|U(Q, 0)|^{2} \widetilde{T}^{2}\left(\frac{k x}{f}-Q\right)+|V(-Q, 0)|^{2} \widetilde{T}^{2}\left(\frac{k x}{f}+Q\right)\right. \\
& \left.+\left[U(Q, 0) V^{*}(-Q, 0) \widetilde{T}\left(\frac{k x}{f}-Q\right) \widetilde{T}\left(\frac{k x}{f}+Q\right)+\text { c.c. }\right]\right\} .
\end{aligned}
$$

The first and second terms illustrate the two interference patterns created by two stimulated beams, i.e. the signal beam with the transverse wavevector $Q$ and the idler beam with the transverse wavevector $-Q$. The third term shows an additional coherent superposition of the two interferences of the converted beams. In result, the interference pattern can be different from the ordinary one due to the "interference term" in the square brackets of Eq. (52). Only for the normal incidence, $Q=0$, the interference pattern is the same as the ordinary one. Figures 5a and 5b show the density patterns of the one-photon interference by varying the transverse wavevector of the input beam for type I and type II crystals, respectively. In Fig. 5a, the fringes alternate between onset and offset when the transverse wavevector $Q$ of the input field is increased until the two fringes are well apart. The offset is due to the destructive interference of two indistinguishable stimulated beams when they are folded. However, for type II crystal, we put two interference patterns for the signal and idler beams together in Fig. 5b, in which the right part is for the signal field, 
and is stronger than the left part (idler). This corresponds to the measurement when the detection is insensitive to the polarizations. Different from type I crystal, when the transverse wavevector $Q$ of the input field is increased, the bright and dark spots of the patterns exchange alternatively until the two fringes are apart. This feature comes from the incoherent superposition of the two patterns for the signal and idler beams.

We go through a long derivation, using the unitary transformation (24) and the bosonic commutation relation, and obtain the second-order correlation function

$$
\begin{aligned}
G^{(2)}\left(x_{1}, x_{2}\right) & =\left[\left|W_{i}\left(x_{1}, Q\right)\right|^{2}+M_{i}\left(x_{1}, x_{1}\right)\right]\left[\left|W_{s}\left(x_{2}, Q\right)\right|^{2}+M_{s}\left(x_{2}, x_{2}\right)\right] \\
& +\left[W_{i}\left(x_{1}, Q\right) W_{s}\left(x_{2}, Q\right) N_{i s}^{*}\left(x_{1}, x_{2}\right)+\text { c.c. }\right]+\left|N_{i s}\left(x_{1}, x_{2}\right)\right|^{2} \\
& +\delta_{i s}\left\{\left[W^{\star}\left(x_{1}, Q\right) W\left(x_{2}, Q\right) M\left(x_{1}, x_{2}\right)+\text { c.c. }\right]+\left|M\left(x_{1}, x_{2}\right)\right|^{2}\right\} .
\end{aligned}
$$

Again, Eq. (53) can describe two types of crystals. For type I crystal, the subscripts $i$ and $s$ are omitted and $\delta_{i s}=1$, whereas for type II crystal $\delta_{i s}=0$. Similar to Eq. (35), the first term is the product of the two one-photon interferences for the converted beams. The second and the fourth terms exhibit the coupling of the interferences between the stimulated and spontaneous processes. This result is similar to the discussion of the image amplification in the optical parametric amplification in which $W(x)$ describes an amplified image. 23] We note that the interferences coming from the stimulated process are uncorrected in positions. This reflects that the stimulated process does not includes the entanglement in transverse wavevectors for the two converted beams. Therefore, the quantum lithography cannot be achieved in the stimulated parametric amplification by input a coherent beam. In Figs. 6, we plot the density patterns of the two-photon interferences by varying the input direction of the signal field. Figures $6 \mathrm{a}$ and 6b show patterns of $G^{(2)}(x, x)$ and $G^{(2)}(x,-x)$ for type I crystal, respectively. These two patterns are similar to the one-photon interference case with the exception of that the sub-wavelength fringe coming from the spontaneous process appears in the central part. In Fig. 6a, the fringe of the signal part (the right side) is stronger than the idler part (the left side), whereas in Fig. 6b, the pattern is mirror-symmetry since exchange of two detectors at a pair of symmetric positions makes no difference. However, Figs. 6c and 6d show patterns of $G^{(2)}(x, x)$ and $G^{(2)}(x,-x)$ for type II crystal, respectively. Correspondingly, Fig. $6 \mathrm{c}$ is similar to the one-photon interference case with the exception of that of the spontaneous contribution. In Fig. 6d, we define $x$ as the transverse position for the detector sensitive to the polarization of the signal beam, so that it is comparable to the one-photon case for the signal beam (the right part in Fig. 5b). Furthermore, in Fig. 6d, the spontaneous process contributes a homogeneous background instead of a sub-wavelength fringe in Fig. 6c. If two detectors in the joint-intensity measurement are polarization-insensitive, one observes a symmetric patterns which is the mirror-symmetry of Fig. 6d.

\section{CONCLUSIONS}

In summary, we formulate the first- and second-order correlation functions in the Young's double-slit interference for both spontaneous and stimulated parametric down-conversions. The results reveal the relations between the first- and second-order correlations, and hence it can explain the complementarity of coherence and entanglement. We show that the nonclassical sub-wavelength two-photon interference can occur macroscopically in a general spontaneous parametric process. For a high gain of SPDC, in which the converted beams contain a huge number of photons, the sub-wavelength interference pattern is intensive with a substantial visibility. This makes the quantum lithography technology in practicability. Moreover, we find an alternative way to observe the sub-wavelength interference for only type I crystal, in which a joint-intensity measurement is performed by a pair of one-photon detectors placed at the symmetric positions. The advantage of this method is that the quantum lithography in type I of SPDC can be performed by two one-photon detectors instead of a two-photon detector which could be unavailable. Since, this effect occurs only in a higher gain of SPDC, it reflects macroscopically quantum nature for the entangled beams containing a huge number of photons. The two ways of observations compete for the visibility, so that at the low gain of SPDC of type I the interference of the first observation reaches perfectness while the second observation disappears. In the stimulated process, the one-photon and two-photon interference patterns generated by a pair of stimulated down-converted beams are alike. For type I crystal, since two converted beams are polarization-indistinguishable, the coherent superposition of two converted beams causes a secondary interference which may fade the fringe when two beams are not well apart. However, this effect does not exist for type II crystal because of the distinguishability in polarization for the two stimulated beams. 


\section{ACKNOWLEDGMENT}

This research was supported by the National Program of Fundamental Research No. 2001 CB309310 and the National Natural Science Foundation of China, Project Nos. 10074008, 60278021 and 10174007.

[1] D. V. Strekalov, A. V. Sergienko, D. N. Klyshko, and Y. H. Shih, Phys. Rev. Lett. 74, 3600 (1995).

[2] P. H. S. Ribeiro, S. Pádua, J. C. M. da Silva, and G. A. Barbosa, Phys. Rev. A 49, 4176 (1994).

[3] P. H. S. Ribeiro and G. A. Barbosa, Phys. Rev. A 54, 3489 (1996).

[4] G. A. Barbosa, Phys. Rev. A 54, 4473 (1996).

[5] E. J. S. Fonseca, C. H. Monken, and S. Pádua, Phys. Rev. Lett. 82, 2868 (1999).

[6] E. J. S. Fonseca, C. H. Monken, S. Pádua, and G. A. Barbosa, Phys. Rev. A 59, 1608 (1999).

[7] E. J. S. Fonseca, P. H. S. Ribeiro, S. Pádua, and C. H. Monken, Phys. Rev. A 60, 1530 (1999); E. J. S. Fonseca, Z. Paulini, P. Nussenzveig, C. H. Monken, and S. Pádua, Phys. Rev. A 63, 043819 (2001)

[8] B. E. A. Saleh, A. F. Abouraddy, A. V. Sergienko, and M. C. Teich, Phys. Rev. A 62, 043816 (2000).

[9] M. D'Angelo, M. V. Chekhova, and Y. Shih, Phys. Rev. Lett. 87, 013602 (2001).

[10] A. F. Abouraddy, M. B. Nasr, B. E. A. Saleh, A. V. Sergienko, and M. C. Teich, Phys. Rev. A 63, 063803 (2001).

[11] A. F. Abouraddy, B. E. A. Saleh, A. V. Sergienko, and M. C. Teich, J. Opt. B: Quantum Semiclass. Opt. 3, S50 (2001).

[12] S. P. Walborn, M. O. T. Cunha, S. Pádua, and C. H. Monken, Phys. Rev. A 65, 033818 (2002).

[13] R. Shimizu, K. Edamatsu, and T. Itoh, Phys. Rev. A 67, 041805 (2003).

[14] G. Brida, E. Cagliero, G. Falzetta, M. Genovese, M. Gramegna, and E. Predazzi, Phys. Rev. A 68, 033803 (2003).

[15] A. Gatti, E. Brambilla, and L. A. Lugiato, Phys. Rev. Lett. 90, 133603 (2003).

[16] J. Jocobson, G. Björk, I. Chuang, and Y. Yamamoto, Phys. Rev. Lett. 74, 4835 (1995).

[17] A. N. Boto, P. Kok, D. S. Abrams, S. L. Braunstein, C. P. Williams, and J. P. Dowling, Phys. Rev. Lett. 85, 2733 (2000).

[18] E. M. Nagasako, S. J. Bentley, R. W. Boyd, and G. S. Agarwal, Phys. Rev. A 64, 043802 (2001).

[19] K. Edamatsu, R. Shimizu, and T. Itoh, Phys. Rev. Lett. 89, 213601 (2002).

[20] M. I. Kolobov and L. A. Lugiato, Phys. Rev. A 52, 4930 (1995).

[21] I. V. Sokolov, M. I. Kolobov, and L. A. Lugiato, Phys. Rev. A 60, 2420 (1999).

[22] E. Brambilla, A. Gatti, M. Bache, and L. A. Lugiato, arXiv:quant-ph/0306116 (2003).

[23] K. Wang, G. Yang, A. Gatti, L. A. Lugiato, J. Opt. B: Quantum Semiclass. Opt. 5, S1 (2003).

[24] A. Gatti, E. Brambilla, L. A. Lugiato, and M. I. Kolobov, Phys. Rev. Lett. 83, 1763 (1999).

Captions of Figures :

Fig. 1. Schemes of Young's double-slit interference with a convex lens: (a) a one-photon (two-photon) detector measures one-photon (two-photon) intensity distribution; two one-photon detectors measure joint-intensity distribution at a pair of symmetric positions. (b) for type II crystal, two-photon intensity distribution is measured by a polarizing beamsplitter (PBS) and two one-photon detectors.

Fig. 2. One-photon (solid line) and two-photon (dashed line) double-slit interference patterns for a coherent beam.

Fig. 3. Visibilities versus the gain of SPDC for different collinear phase-mismatching (a) $\Delta_{0}=-5.85$; (b) $\Delta_{0}=0$; and (c) $\Delta_{0}=5.85$. Solid and Dashed lines designate $\mathcal{V}_{1}$ for type II and type I crystals, respectively; Dotted line designates $\mathcal{V}_{2}$ for type I crystal.

Fig. 4. Two-photon interference patterns versus the normalized bandwidth of SPDC $q_{0} b /(2 \pi)$ : (a) $G^{(2)}(X, X)$ for type I crystal; (b) $G^{(2)}(X,-X)$ for type I crystal; (c) $G^{(2)}(X, X)$ for type II crystal; and (d) $G^{(2)}(X,-X)$ for type II crystal, where $X=x k b /(2 \pi f)$ is the normalized transverse position in the detection plane. The gains are set as $g=(1 / 2) \log 1.5$ in (a) and (c), and $g=(1 / 2) \log 10$ in (b) and (d). The other parameters in Figs. 4-6 are taken as the phase matching $\Delta_{0}=0$, the ratio $b / d=0.2$, and $\frac{k b \Omega_{0}}{4 \pi^{2} f}=1$ for an arbitrary unit of the correlation functions.

Fig. 5. Stimulated one-photon interference patterns by varying the normalized transverse wavevector of the input field $Q b /(2 \pi)$ for (a) type I crystal and (b) type II crystal. The normalized bandwidth and the input intensity are taken as $q_{0} b /(2 \pi)=2$ and $\frac{2 k b^{2}}{\pi f} A^{2}=1$, respectively.

Fig. 6. Stimulated two-photon interference patterns by varying the normalized transverse wavevector of the input field $Q b /(2 \pi)$ : (a) $G^{(2)}(x, x)$ for type I crystal; (b) $G^{(2)}(x,-x)$ for type I crystal; (c) $G^{(2)}(x, x)$ for type II crystal; and $(\mathrm{d}) G^{(2)}(x,-x)$ for type II crystal. The normalized bandwidth and the input intensity are the same as in Fig. 5 . 


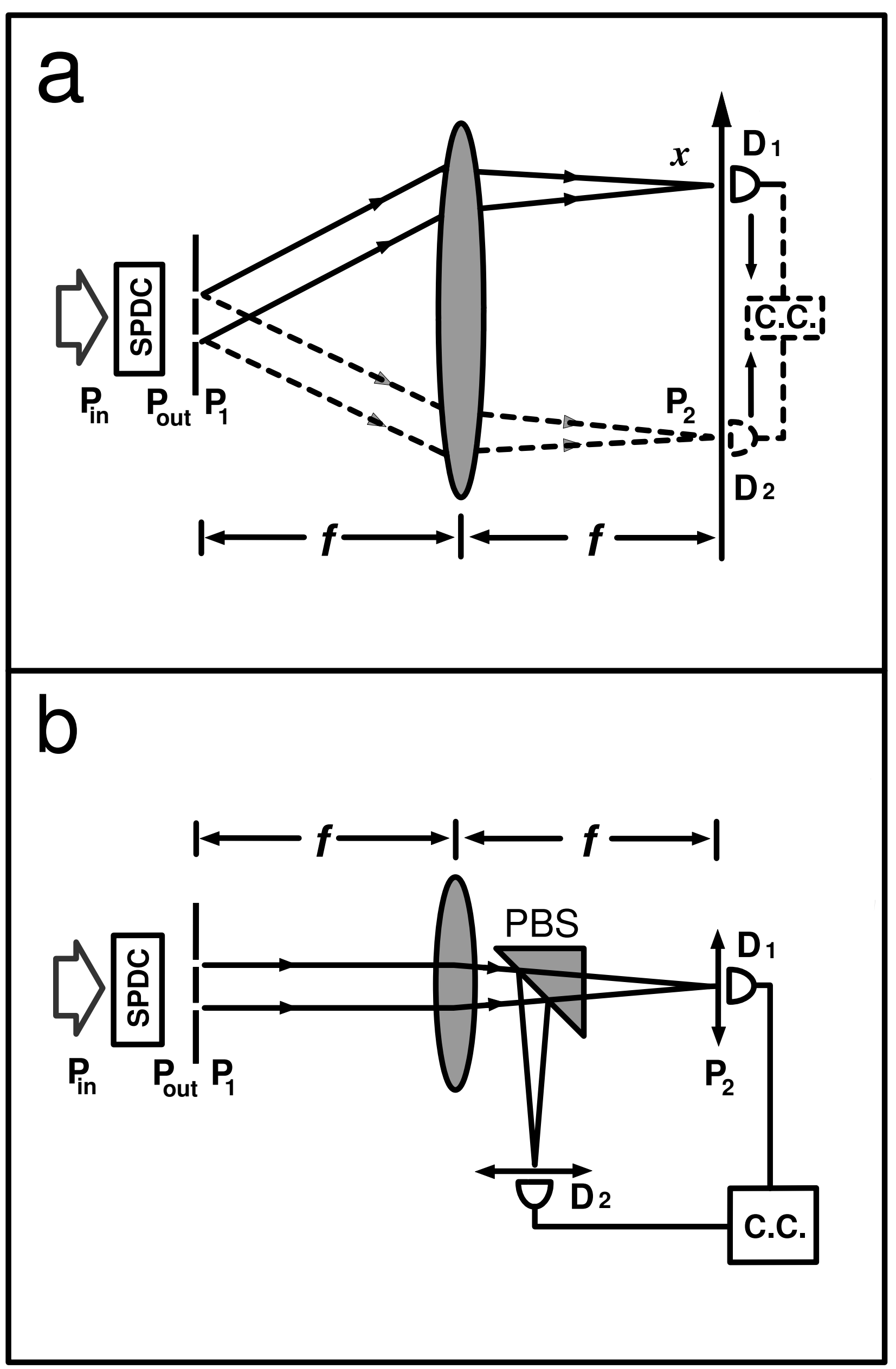




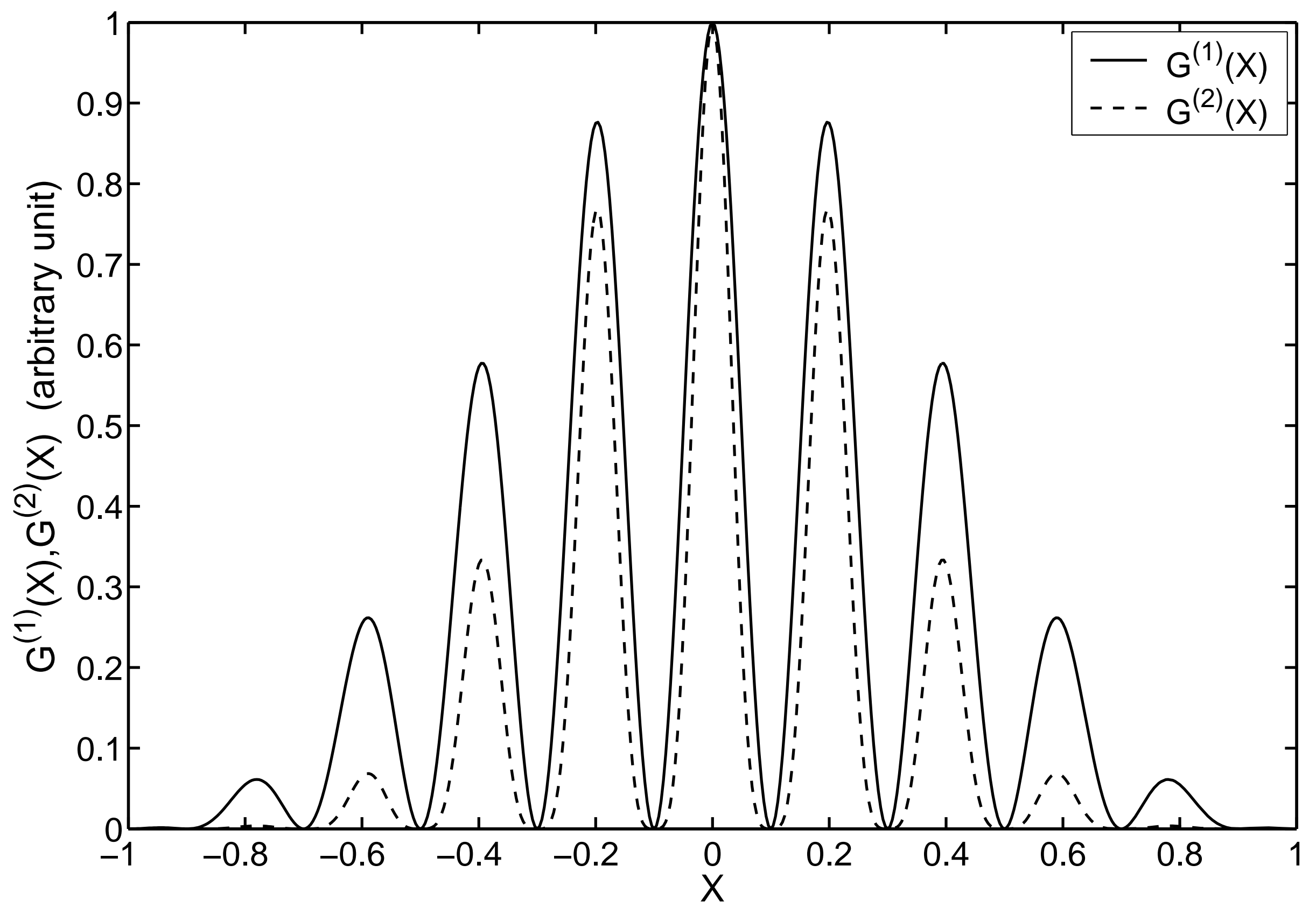



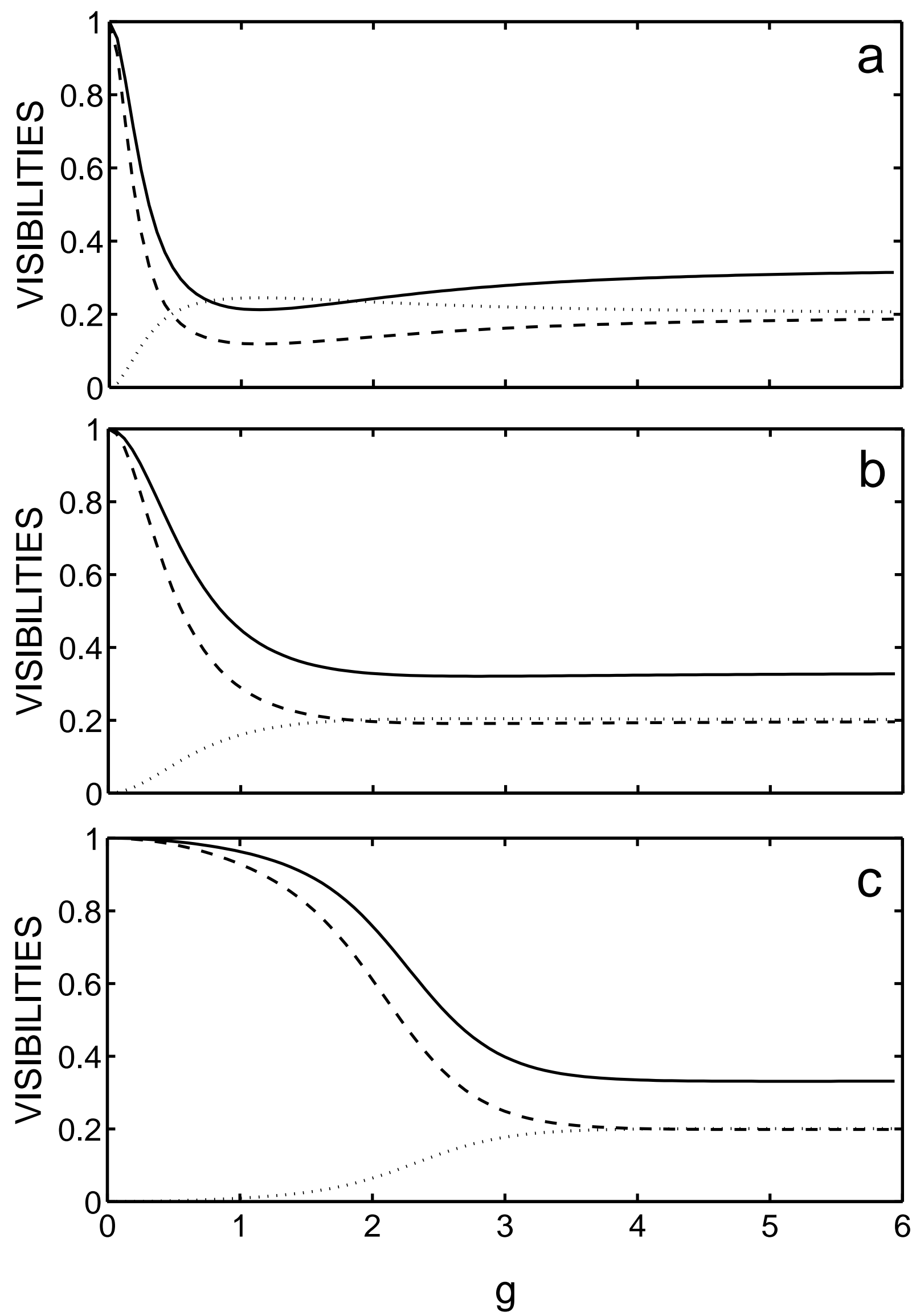

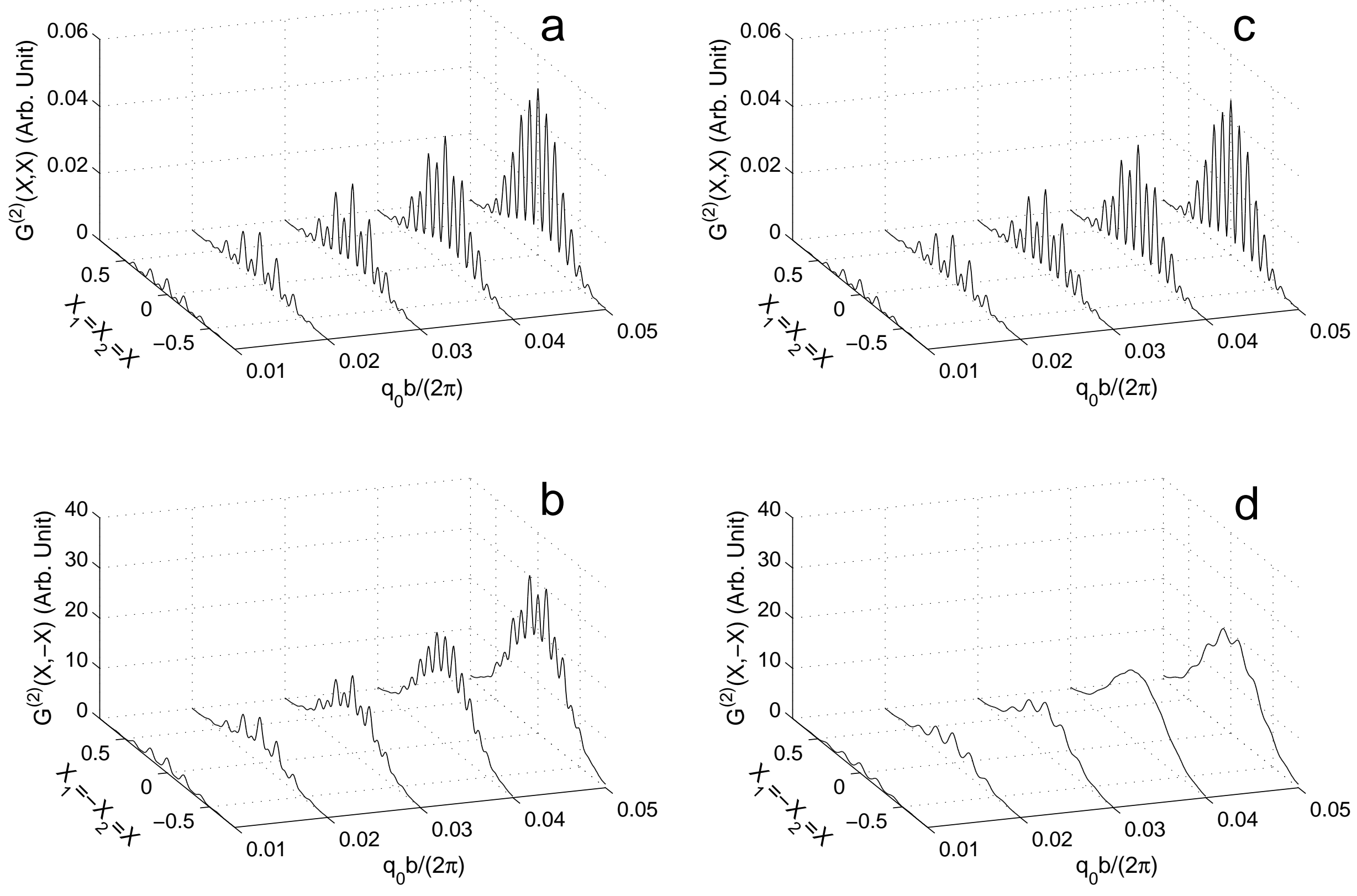


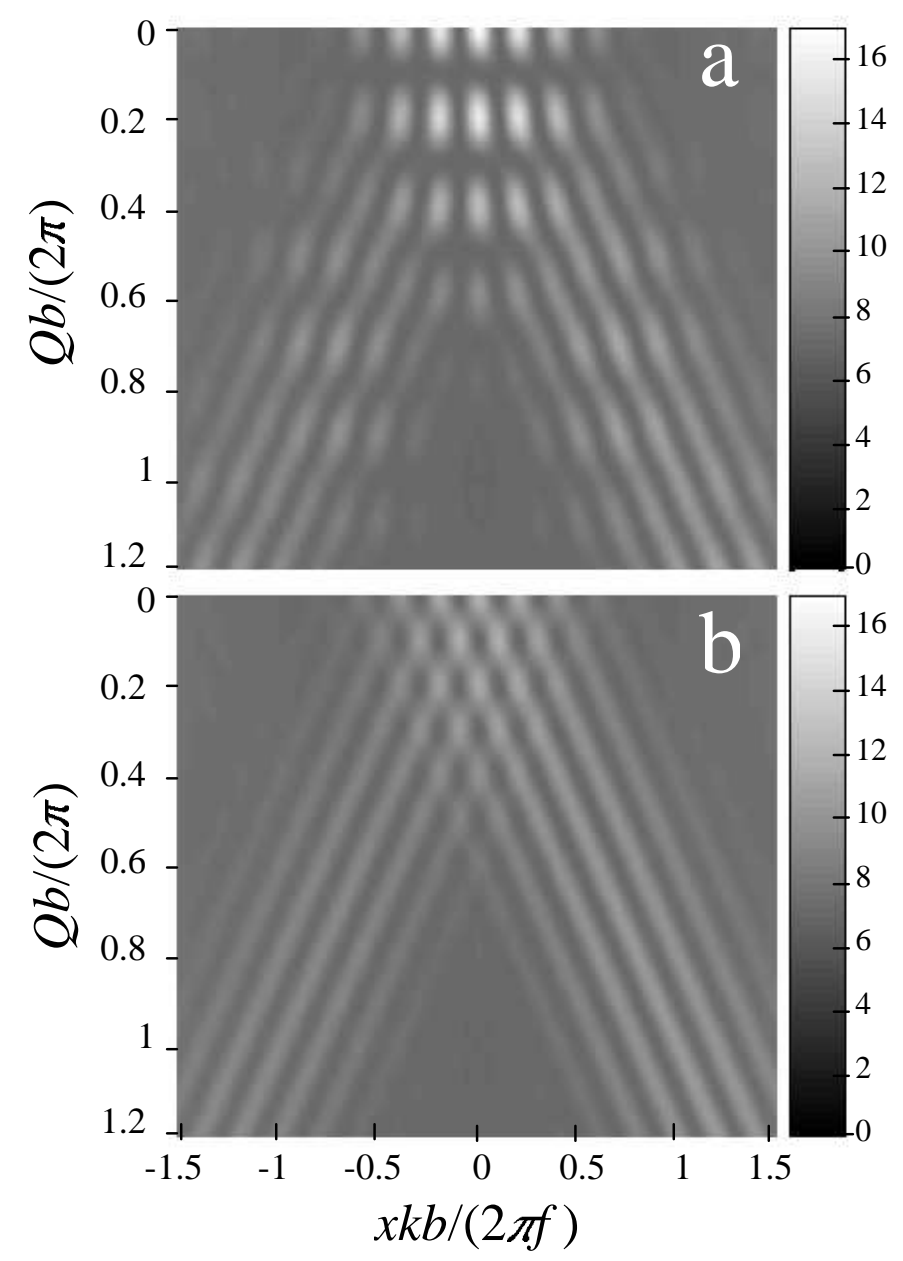




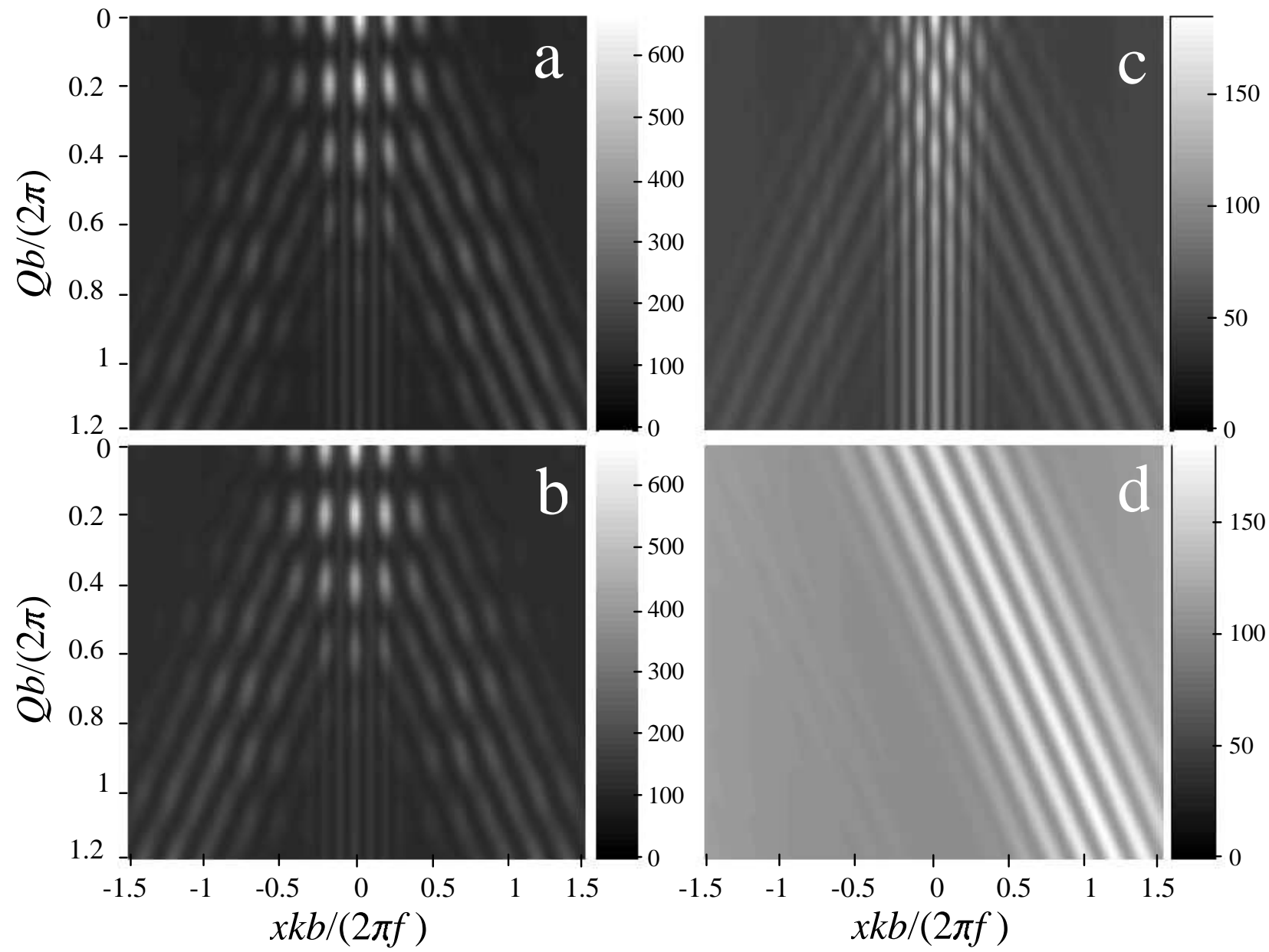

